\title{
Influence of the number of re-emitters on a diffuser modelling for the optimization stage
}

\section{Influencia del número de elementos reemisores en el modelado de difusores durante la etapa de optimización}

\author{
P. Blanco ${ }^{1 *}$, J. Arasa ${ }^{2, S}$ \\ 1. Simulacions Optiques S.L. (Snelloptics) C/Sant Quirze 91, 5-2, E-08221 Terrassa \\ 2. Centro de Desarrollo de Sensores, Instrumentación y Sistemas (CD6) de la Universidad \\ Politécnica de Cataluña, Rambla Sant Nebridi 10, E-08222 Terrassa \\ ${ }^{(*)}$ E-mail: tricia.blanco@snelloptics.com \\ $S:$ SEDOPTICA member \\ Received: 26/03/2015 Accepted: 10/05/2016 \\ DOI: $10.7149 /$ OPA.49.2.48506
}

\begin{abstract}
:
Inside the process of optical design, the diffusion phenomenon is usually set apart until the final stage: the study of tolerances. So, its influence is evaluated considering the whole of the system after having designed it taking into account all other parameters. The phenomenon of diffusion is relevant in many optical designs and its influence can determine the final design. So, this procedure, relegating diffusion to stage study, does not means that its influence over the system isn't heavy, but stems from the high computational cost of considering it on earlier stages. But there is a way to include it before the tolerances study stage by using a dual optimization process with an on/off mechanism. Thus the best system configuration is obtained considering the diffusion. In order to reduce the computation time we adjust dynamically the values of the diffuser model control parameters. The number of re-emitters is one of these strategic control parameters, inside this parameter are included two different values, the number of reemitting focus and the number of reemitting rays considered. Varying this value, different degrees of model accuracy can be obtained, allowing to adapt the model accuracy depending on the merit function instantaneous value. In this paper we analyse the use of a diffuser model, varying the parameter of the number of reemitters, and its influence on optical system computer aided design (CAD). We make so by employing a simple system consisting on a light source, a biconvex lens, on which the diffusion model is applied only on the second surface, and a detection display.
\end{abstract}

Key words: optical design, scattering, stray light, optical diffusion, ray tracing, surface modelling, optimization

\section{RESUMEN:}

En el marco de trabajo del diseño óptico, el fenómeno de la difusión normalmente queda relegado a la fase final del estudio de tolerancias, momento en el que se valora su influencia en el sistema ya diseñado. El hecho de relegar la difusión a la etapa de estudio no es indicador de que no pueda influir en el diseño, sino del alto coste computacional que implicaría su inclusión en etapas anteriores. En múltiples diseños ópticos resulta relevante el fenómeno de la difusión y su influencia puede determinar cómo se realiza el diseño. Una manera de incluirlo en las etapas previas a la etapa de estudio de tolerancias es mediante el uso de un proceso de optimización dual con un mecanismo de transición que activa/desactiva el modelo de difusión dentro del propio proceso. De esta manera se obtiene la mejor configuración teniendo en cuenta la difusión que presenta el sistema. Una manera de reducir el tiempo de cómputo consiste en adaptar los valores de los parámetros de control del modelo de difusor dinámicamente siendo uno de los parámetros estratégicos el número de elementos de reemisión, este parámetro está formado por el número de focos de reemisión y el número de rayos que se consideran en el modelo. Mediante la variación de este valor se obtienen diferentes grados de precisión del modelo, lo que nos permite adaptarlo al proceso de optimización, dependiendo del valor instantáneo de la función de mérito utilizada. En el presente trabajo se realiza un análisis de la influencia del número de elementos de reemisión de un modelo de difusión dentro del marco del diseño asistido por computador (CAD) empleando para ello un 
sistema sencillo que consta de una fuente de iluminación, una lente biconvexa, sobre la que se aplica la estrategia de inclusión de los difusores únicamente en la segunda superficie, y una pantalla de detección.

Palabras clave: diseño óptico, scattering, stray light, difusión óptica, ray tracing, modelo superficial, optimización

\section{REFERENCES AND LINKS / REFERENCIAS Y ENLACES}

[1] W. J. Smith, Modern Lens Design, Second Edition, McGraw-Hill (1992)

[2] J. Macdonald, P. Mouroulis, Geometrical Optics and Optical Design, Oxford University Press (1997)

[3] R.R. Shannon, The Art and Science of optical design, Cambridge University Press (1997) http://dx.doi.org/10.1017/CB09780511816529

[4] R.P. Breault, M. Turner, "Optical Design and Stray Light Concepts and Principles", Springer Handbook of Lasers and Optics, pp 427-445 (2012) http://dx.doi.org/10.1007/978-3-642-19409-2_7

[5] Y. Chen, C.H. Hu, Y.C. Luo, P. Wang, "Lens Design of Low Light Level Night Vision Objective Optical System", Appl. Mech. Mat. 568-570, 1778-1781, (2014) http://dx.doi.org/10.4028/www.scientific.net/AMM.568-570.1778

[6] P.A. Lightsey ; Z. Wei; "James Webb Space Telescope stray light performance status update". Proc. SPIE 8442, Space Telescopes and Instrumentation: Optical, Infrared, and Millimeter Wave, 84423B (2012)

[7] D. Cheng, Y. Wang, L. Yu, X. Liu, "Optical design and evaluation of a $4 \mathrm{~mm}$ cost-effective ultrahigh-definition arthroscope", Biomed. Opt. Express 5, 2697-2714 (2014) http://dx.doi.org/10.1364/BOE.5.002697

[8] G.L. Peterson, "Stray light calculation methods with optical ray trace software", Proc. SPIE 3780 , Optical Design and Analysis Software, 132, (1999)

[9] M. Born, E. Wolf, Principles of Optics, Cambridge University Press (1999)

[10] R.N. Pfisterer, "Approximated Scatter Models for Stray Light Analysis", OPN Optics \& Photonics News, OSA, 16-17, October (2011)

[11] H.J. Frasch, G. Steinmetz, "Modelling surface and volume scattering with raytracing software", Proc. SPIE 5962, Optical Design and Engineering II, 59620D ( 2005) http://dx.doi.org/10.1117/12.624944

[12] S.S. Steadman, B.K. Likeness, "GUERAP III simulation of stray light phenomena”, Proc SPIE 107, (1977)

[13] B. M. Boyce, "Guerap II- A Computer Program For The Analysis Of The Stray Radiation Rejection Capabilities Of Optical Systems", Proc SPIE 0107, Stray Light Problems in Optical Systems, (1977)

[14] C. Pizarro, Desarrollo de un proceso de optimización adaptado a sistemas ópticos con detectores acoplados, Tesis Doctoral, UPC (2002)

[15] P. Blanco, C. Pizarro, J. Arasa, "Optimizaciones duales para procesos de gran coste computacional. Ensayo: minimización de spot y ghost en un sistema óptico sencillo", OPTOEL'09 


\section{Introducción}

El proceso clásico de diseño óptico consta de diversas etapas [1, 2, 3]. En cada una de ellas se incluyen una serie de características mediante las cuales el diseño se acerca paulatinamente al comportamiento real. No obstante, existen algunos fenómenos como los de difusión o la difracción que, aunque para ciertas aplicaciones pueden ser clave, no es posible incluir hasta que el diseño se considera estable. Esto se debe a que el tiempo de cómputo hace inviable poder considerarlos en etapas anteriores a la de estudio de tolerancias [4]. El alto tiempo de cómputo no sólo es debido al modelo de difusión en sí, sino también al hecho de que para que los resultados sean fiables, la simulación del sistema debe ser no secuencial. Es decir, resulta imprescindible que los elementos no se analicen según un orden preestablecido, se incluyan las monturas del sistema y que además se consideren los balances de energía de manera ajustada.

Dependiendo de la aplicación concreta, el resultado del estudio de tolerancias de difusión puede provocar el rediseño completo del sistema al no cumplir las especificaciones de luz parásita o stray light. Este sería el caso de aplicaciones en las que se deben detectar valores muy pequeños de energía como en el diseño de ópticas para visión nocturna [5], el diseño de telescopios [6] o el diseño de sensores para detección de luz difundida por tejidos biológicos en aplicaciones biomédicas [7]. En otros casos, se podría hacer que la etapa de optimización sea más corta relajando las restricciones que impone el criterio de calidad sobre el sistema óptico. Ese es el caso de las aplicaciones de iluminación que hacen uso de elementos difusores como partes activas del diseño para uniformizar la distribución de iluminación resultante. En ambos casos, la inclusión de la difusión en la etapa de optimización del diseño sería de gran ayuda.

\section{Especificaciones del modelo difusor}

El modelo de difusor que se ha utilizado en el presente trabajo se ha diseñado para su uso mediante la técnica de trazado de rayos o ray tracing. Esta técnica es de uso habitual en el diseño de sistemas ópticos [8]. Para ello se ha considerado que el modelo se aplicará en un entorno de diseño asistido por computador (CAD) sobre una geometría independiente del modelo. La difusión así tratada equivale a superponer una capa de comportamiento difusor sobre la superficie a considerar, lo que nos permitirá activar o desactivar la propiedad de la superficie según convenga. En la figura 1 se muestra un detalle del funcionamiento de diferentes capas que pueden superponerse sobre una superficie. La capa 1 es la capa geométrica (el plano se puede substituir por otra geometría), la capa 2 es la capa de interacción primaria donde se aplican las leyes de Snell y Fresnel y la capa 3 es la capa de interacción secundaria donde se aplica el modelo de difusión. Un ejemplo de dicho funcionamiento puede verse en esta misma figura, donde la longitud de los rayos reemitidos representa la cantidad de intensidad radiante asociada a cada uno de ellos.

\begin{tabular}{|l|r|r|r|}
\hline Caso 1 & CAPA 1 & CAPA 2 & CAPA 3 \\
\hline Caso 2 & & & \\
\hline Caso 3 & & & \\
\hline
\end{tabular}

Fig. 1. Esquema de funcionamiento por capas para tres casos: interacción primaria de refracción y reflexión (caso 1), interacción primaria sólo refracción (caso 2) e interacción primaria sólo reflexión (caso 3).

El flujo transportado por cada rayo que llega a la superficie, y que genera un foco de reemisión de difusión, debe conservarse al aplicar el principio de conservación de la energía [9]. La forma en que se pesan los rayos reemitidos generados por difusión y el flujo asociado a cada uno de ellos se ve afectado por este principio. Este balance energético se realiza teniendo en cuenta la contribución de cada uno de estos rayos (ecuación 1)

$$
\Phi_{i}=\Phi_{0} \cdot \frac{f(\theta, \varphi) \cdot \omega_{i}}{\sum_{4 \pi} f(\theta, \varphi) \cdot \omega_{j}}
$$


Donde $\Phi_{0}$, es el flujo a repartir, $\Phi_{i}$ el flujo asignado al rayo reemitido i y $f(\theta, \varphi)$ la función de distribución energético-espacial. En la misma ecuación $\theta$ se define como el ángulo respecto de la normal de la superficie, $\varphi$ es el ángulo en el plano tangente a la superficie, $\omega_{\mathrm{i}}$ es el ángulo sólido asignado al rayo reemitido i y el sumatorio del denominador es el factor de normalización.

El modelo también debe poder considerar diferentes funciones de distribución energético-espacial $\mathrm{f}(\theta, \varphi)$. Estas funciones describen la fenomenología de la propiedad difusora [10], siendo dos de las funciones de distribución más usuales la del difusor ideal o lambertiano y la del modelo ABg. El modelo de difusor ideal es un modelo puramente teórico $[9,10]$ y el modelo $\mathrm{ABg}$ se utiliza en diseño óptico para caracterizar superficies de pulido óptico [10] de diferentes elementos ópticos como lentes, espejos y prismas entre otros.

En la ecuación 2 se describe la función de distribución para un difusor ideal [9] con simetría de revolución en torno a la normal a la superficie y radiancia uniforme sobre la superficie

$$
\Phi_{i}=\Phi_{0} \cdot N o r m \cdot \cos \left(\theta_{i}\right) \cdot \omega_{i}
$$

Siendo $\Phi_{i}$ el flujo que se asigna al rayo reemitido i, $\Phi_{0}$ el flujo a repartir, Norm el factor de normalización, $\theta_{\mathrm{i}}$ se define como el ángulo entre la dirección del rayo de reemisión y la normal de la superficie y $\omega_{\mathrm{i}}$ es el ángulo sólido asignado al rayo reemitido i.

En el caso del cálculo del modelo ABg [10] (ecuación 3) se utiliza el ángulo que forman las direcciones del rayo generador del foco de reemisión y la del rayo de reemisión proyectadas en el plano tangente a la superficie difusora $\left(\beta_{0}\right.$ y $\beta_{\mathrm{i}}$ respectivamente).

$$
\Phi_{i}=\Phi_{0} \cdot N o r m \cdot \frac{\mathrm{A}}{B+\left|\beta_{i}-\beta_{0}\right|^{g}} \cdot \omega_{i}
$$

En la que $\Phi_{i}$ es el flujo que se asigna al rayo reemitido i, $\Phi_{0}$ es el flujo a repartir, Norm el factor de normalización. $\beta_{0}$ y $\beta_{\mathrm{i}}$ son los ángulos de las direcciones del rayo incidente y del rayo reemitido i en el plano tangente a la interacción. A, B y g son los parámetros propios del modelo y $\omega_{\mathrm{i}}$ es el ángulo sólido asignado al rayo reemitido $\mathrm{i}$.

Para una correcta descripción de la distribución energético-espacial es necesario el cálculo previo de las direcciones de reemisión de los rayos que se generan durante la difusión, es decir de los rayos reemitidos. La dirección de propagación de estos rayos se genera mediante una función de distribución espacial que puede depender de la naturaleza de la superficie. Un método habitual para el cálculo de la dirección de reemisión es el de Monte Carlo[11, 12,13]. No obstante, este método presenta el grave inconveniente de necesitar de un elevado número de elementos para poder obtener suficiente precisión de cálculo. Otra solución que se utiliza en sistemas de cálculo de análisis de stray light es el uso del llamado 'importance sampling'[12]. Este método limita el ángulo de reemisión del foco a partir del ángulo de visión del detector. También es posible calcular las direcciones de reemisión de los diferentes rayos mediante el uso de una matriz que ocupa el ángulo sólido de reemisión. En este tercer caso el principal inconveniente es el posible aliasing y consecuentemente la aparición de falsas estructuras[14]. Una vez conocidas las direcciones de reemisión y asignado el ángulo sólido equivalente para cada rayo reemitido se calcula el flujo de cada rayo aplicando la ecuación (1).

Las especificaciones que se requieren del modelo nos permiten poder acoplarlo fácilmente a un proceso de optimización dual [15]. En la figura 2, se muestra el diagrama de bloques de dicho proceso. El hecho de considerar las propiedades difusoras como una capa facilita la activación o desactivación de las mismas. Los parámetros de control del modelo de difusor nos ayudarán a reducir el tiempo de cómputo de la etapa de optimización con las propiedades de difusión activas. 


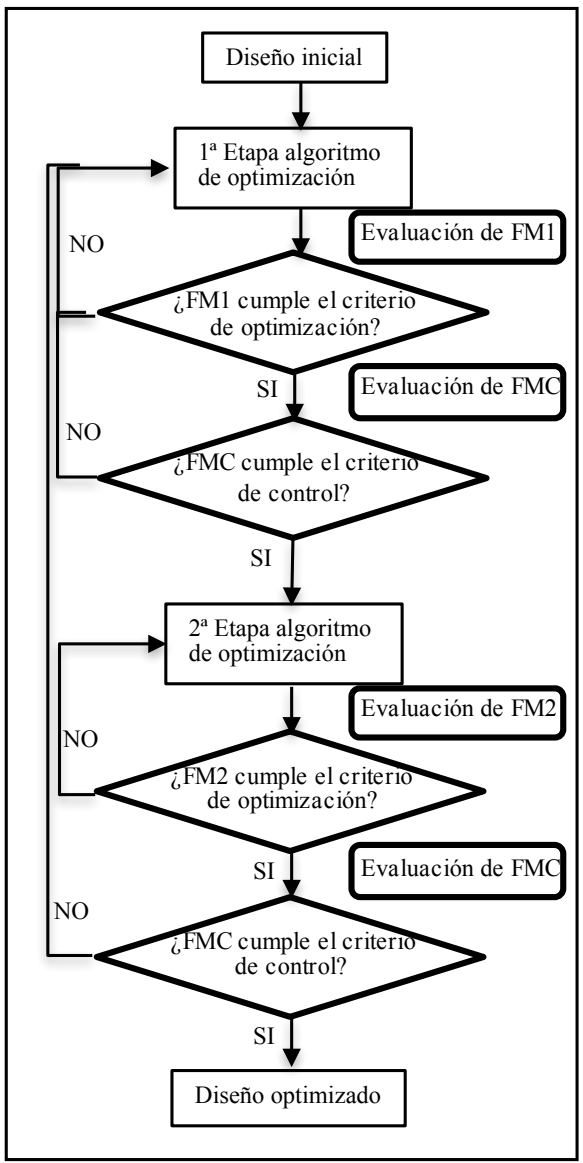

Fig 2. Diagrama de bloques de optimización dual. FM1 y FM2 son la función de mérito y FMC es la función de mérito de control que activa/desactiva el modelo de difusión para la $2^{a}$ etapa del algoritmo de optimización.

\section{Parámetros de control del modelo difusor}

Una vez especificado el modelo de difusor que será utilizado, es necesario definir los parámetros de control que se emplearán para activar/desactivar el modelado del difusor en la etapa de optimización. Estos parámetros se utilizan para ajustar el delicado equilibrio entre la precisión del cálculo de los efectos de las propiedades de difusión por un lado y, por otro, un tiempo de cómputo que sea razonable para la etapa de diseño en curso. Para la etapa de estudio de tolerancias, la precisión del modelo puede ser alta sin perjudicar el tiempo de cómputo. No obstante, durante la etapa de optimización, que actualmente consiste en un proceso semiautomático, el número de sistemas que se calculan es elevado, por lo que resulta crítico que estos parámetros puedan ser recalculados por el propio proceso de optimización.

Los parámetros seleccionados para el control del modelo de difusión son: el número de elementos de reemisión, el ángulo de interés de reemisión y el límite inferior de cantidad de flujo del sistema.

\section{3.a. Número de elementos de reemisión}

Uno de los parámetros de control que regulan la precisión del modelo es el número de elementos de reemisión. Estos elementos son los focos de reemisión y los rayos de reemisión. Las características de dirección y flujo de los primeros, los focos de reemisión, se obtienen a partir de la capa 2 del modelo descrito en la (figura 1). Los segundos, los rayos de reemisión, se generan en cada uno de los focos de reemisión aplicando la ecuación concreta del modelo de difusión.

El valor del número de focos de reemisión (N) está relacionado con la manera en cómo se muestrea la fuente de emisión y las interacciones previas del sistema óptico. Se trata de un valor de difícil control ya que, en el caso de difusión por reflexión el valor de $\mathrm{N}$ se incrementa dinámicamente.

El valor del número de rayos de reemisión (n) es más fácil de controlar. Este valor afecta a la precisión del modelo de difusión que se aplica en cada foco de reemisión y determina el valor de ángulo sólido asociado a cada uno de los rayos de reemisión. Para un ángulo sólido total de reemisión de $\Omega$ 
estereorradianes, considerando una distribución uniforme de los rayos de reemisión, a cada uno de ellos le corresponde un ángulo sólido $\omega_{\mathrm{i}}$ según la ecuación 4.

$$
\omega_{i}=\frac{\Omega}{n}
$$

Este valor $\mathrm{n}$ también repercute en el valor de flujo de cada uno de los rayos de reemisión a través de la función de distribución energético-espacial. En la figura 3 se han esquematizado los ángulos sólidos para diferentes valores de $n$.

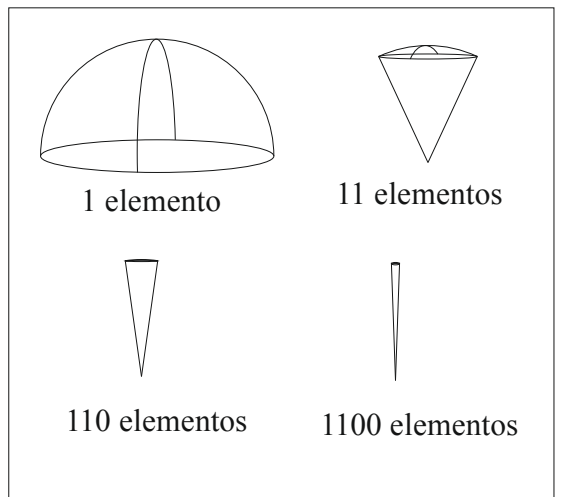

Fig 3. Representación del ángulo sólido asociado a cada rayo para cuatro valores de $\mathrm{n}$ cuando se aplica a un ángulo sólido de $2 \pi$ sr.

Al aumentar el valor de n, los valores de flujo asociados a cada ángulo sólido serán más precisos, y por lo tanto su comportamiento más cercano a la realidad. No obstante, un valor excesivamente elevado comporta el inconveniente de aumentar sustancialmente el tiempo de cálculo, ya que por cada foco de reemisión se generan n nuevos rayos de reemisión. Como resultado, el crecimiento de la carga computacional es de tipo potencial respecto al número de focos de reemisión $\mathrm{N}$.

\section{3.b. Ángulo sólido de interés de reemisión}

Además del número de rayos de reemisión, el modelo difusor también tiene como parámetro de control el ángulo sólido de interés de reemisión. Este parámetro indica el ángulo sólido activo que el diseñador considera en el reparto de flujo del foco de reemisión. El valor de este parámetro nos da la proporción de flujo del foco que se reemitirá durante el cálculo debido a que el flujo del foco se reparte según la función de distribución energético-espacial para todo el espacio. Una consecuencia del uso de este parámetro de control está en el balance energético del sistema óptico en su conjunto ya que no se reemite la totalidad del flujo recibido en cada foco de reemisión. Dependiendo de la función de distribución energético-espacial este valor será mayor o menor y puede afectar a la distribución de flujo que llega al detector si tenemos en cuenta la contribución de este efecto para un número elevado de rayos con valores pequeños de flujo.

En la tabla I se presenta la proporción de flujo que se desprecia en el caso de un difusor ideal para 5 valores diferentes de ángulo solido de interés.

TABLA 1. Porcentaje de flujo de un difusor ideal que no se reemite para diferentes valores de ángulo sólido de interés. El ángulo sólido se presenta como el porcentaje sobre la esfera, el valor de ángulo plano para un casquete esférico equivalente y su valor absoluto.

\begin{tabular}{|c|c|c|c|}
\hline \hline \multicolumn{3}{|c|}{ ÁNGULO SóLIDO DE INTERÉS } & \multirow{2}{*}{ \% FLUJO NO REEMITIDO } \\
\hline \hline$\%$ & Angulo plano $\left(^{\circ}\right)$ & Ángulo sólido (sr) & 0,50 \\
$75 \%$ & 84,27 & 6,22 & 3,17 \\
$60 \%$ & 75,53 & 5,78 & 8,34 \\
$45 \%$ & 66,44 & 5,28 & 16,48 \\
$30 \%$ & 56,64 & 4,38 & 28,56 \\
\hline \hline
\end{tabular}

\section{3.c. Límite inferior de cantidad de flujo}

Como se ha visto en el apartado 3.a, el valor del número de rayos de reemisión determina el valor del ángulo sólido asociado a cada uno de ellos y, mediante la función de distribución energético-espacial, influye en la distribución de flujo. Cuando este valor es elevado o para ciertos valores de ángulo sólido el valor del flujo puede ser muy pequeño. Ahora bien, estas contribuciones de valor bajo de flujo no 
se deben menospreciar a priori ya que también contribuyen en el cálculo del resultado. Es por ello que la activación del modelo de difusión afecta al parámetro de control del sistema que evita que un rayo vea reducida su energía de manera infinita: el límite inferior de cantidad de flujo.

El límite inferior de cantidad de flujo del sistema no sólo afecta al modelo, permitiendo el trazado de rayos con un valor muy pequeño de flujo, sino que además activa los efectos de reflexiones múltiples que, tal vez, no se habían activado todavía. Por tanto, debemos asegurarnos que todos aquellos rayos con valor de flujo del mismo orden que el límite de la precisión del modelo difusor sean incluidos en el cálculo.

\section{Entorno de prueba}

El presente trabajo se centra en el análisis del número de elementos de reemisión y su influencia en la precisión y control del modelo de difusión. El resto de parámetros de control se han fijado de manera que no interfieran en el comportamiento del sistema óptico. Para obtener los datos necesarios y poder analizarlos se ha generado un entorno de prueba. Dicho entorno consta de un sistema óptico, una herramienta de simulación y los elementos de análisis.

\section{4.a. Sistema óptico}

El sistema óptico utilizado se muestra en la figura 4. y consta de una lente biconvexa en cuya segunda cara se aplicará el modelo de difusor, los parámetros de la cual se muestran en la tabla 2, una fuente de iluminación colimada, monocromática $(587,56 \mathrm{~nm})$ e incoherente y una pantalla detectora de $65 \mathrm{~mm}$ de diámetro situada a una distancia de $75.78 \mathrm{~mm}$ de la superficie posterior de la lente.

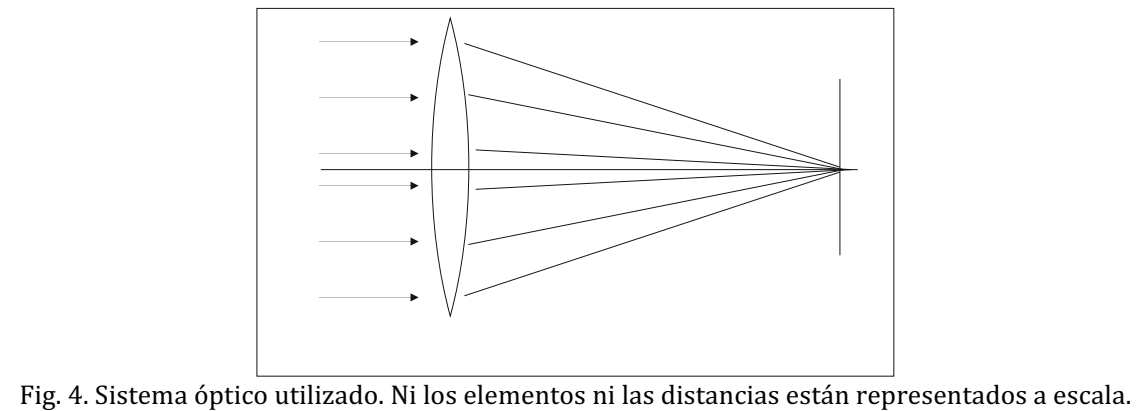

TABLA 2. Valores de los parámetros de la lente biconvexa utilizada

\begin{tabular}{|l|r|}
\hline \hline PARÁMETRO & \\
\hline \hline Material & SK16 \\
Radio primera superficie $(\mathrm{mm})$ & 88,66 \\
Radio primera superficie $(\mathrm{mm})$ & $-108,41$ \\
Diámetro $\quad(\mathrm{mm})$ & 25,00 \\
Grosor de centro (mm) & 45,59 \\
\hline \hline
\end{tabular}

Para la fuente se ha considerado como variable su muestreo, llevándola a dos casos extremos, un solo rayo y un diámetro de $24,5 \mathrm{~mm}$ en la que se ha realizado un muestreo uniforme de lado $11 \mathrm{x} 11$ sobre una superficie cuadrada de $24,5 \mathrm{~mm}$ tal y como se muestra en la figura 5 . En este segundo caso, la $\mathrm{N}$ inicial es 81.

La segunda superficie de la lente se ha caracterizado como superficie difusora por transmisión y reflexión. La función de distribución energético-espacial utilizada se muestra en la ecuación 5. La distribución en $\varphi$ es uniforme para cada $\theta$ y $\theta$ es el ángulo respecto al foco de reemisión, el rayo refractado o reflejado según el caso, y se calcula siguiendo el método de Monte Carlo, asimismo se ha considerado que la superficie absorbe el 5\% del flujo del foco de reemisión.

$$
f(\theta, \varphi)=\left\{\begin{array}{c}
0,6, \quad \theta=0^{\circ} \\
\cos (\theta), \quad 0<\theta<60^{\circ} \\
0, \quad \theta>60^{\circ}
\end{array}\right.
$$




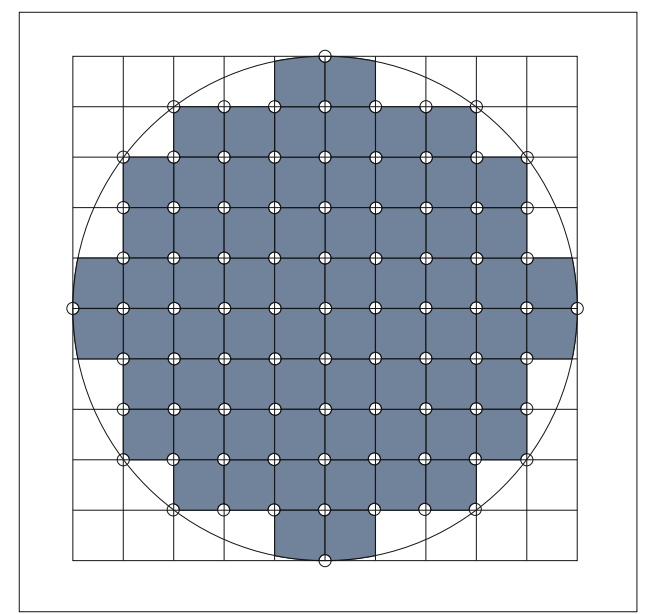

Fig.5. Muestreo de la segunda fuente de iluminación utilizada para el análisis. Los puntos blancos son las posiciones iniciales de los rayos trazados. El diámetro de la circunferencia es de 24,5 mm y la separación entre puntos de 2,45 mm para cada una de las dimensiones.

El resto de parámetros fijados en el ejemplo son:

A) el ángulo sólido de interés de reemisión, fijado en $60^{\circ}$ que corresponde a un $90 \%$ del flujo que llega al foco de reemisión,

B) el límite inferior de cantidad de flujo fijado en $10^{-9}$ u.a., $y$

C) el número máximo de interacciones múltiples sobre la misma superficie fijado en 5 y el número total de interacciones para un rayo fijado en 6.

\section{4.b. Herramienta de simulación}

El sistema se ha simulado en un PC AMD AthlonTM 64x2 Dual Core Processor $5600+2.81 \mathrm{GHz}$ con 1,87GB de RAM y sistema operativo Windows XP SP2 utilizando el software de desarrollo de SnellOptics ${ }^{\mathrm{TM}}$.

\section{4.c. Elementos de análisis}

La influencia del número de elementos de reemisión se ha analizado empleando dos estrategias:

A) incrementando el número de rayos que inciden en la superficie difusora (focos de reemisión), que se refleja en la variación en el muestreo de la fuente de iluminación, y

B) variando el número de rayos de reemisión del modelo difusor.

Y para realizar la valoración de la influencia del número de elementos de reemisión se ha considerado el tiempo empleado en la simulación, el número de interacciones totales del sistema y, de forma cualitativa, la distribución de flujo sobre el plano de detección.

\section{Resultados}

Se ha simulado el sistema óptico empleando las dos aproximaciones extremas de la fuente de luz; o bien empleando un único rayo o bien empleando un muestreo de 11 x11 rayos a través de una pupila dando lugar a $\mathrm{N}=81$.

La primera información relevante se puede ver en las figuras 6 y 7 en las que se representa en el eje de abscisas el número de rayos (n) y en el de ordenadas el número de focos de reemisión $(\mathrm{N})$ autogenerados en el propio proceso de simulación.

La figura 6 se ha iniciado con un muestreo de un único rayo ( $N=1$ ), mientras que en la figura 7 se ha iniciado con un muestro de $\mathrm{N}=81$ rayos, en ambos casos las dos gráficas permiten establecer una relación directa entre $\mathrm{N}$, focos de reemisión del flujo correspondiente y $\mathrm{n}$, rayos que se generan según el modelo de difusión. De su análisis podemos deducir que se puede emplear únicamente n (número de rayos que se generan según el modelo de difusión) para activar/desactivar la función de mérito que incluye la difusión 


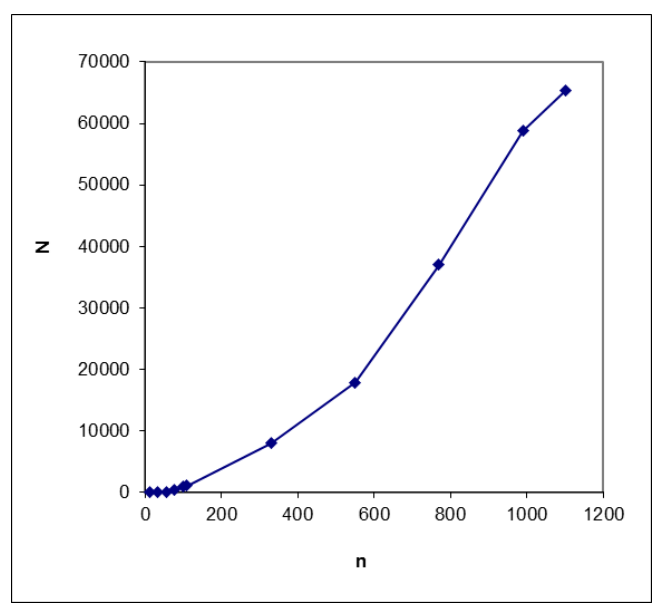

Fig. 6. Relación entre el número de focos de reemisión (N) respecto al número de rayos de reemisión (n), en este caso se ha iniciado la simulación con $\mathrm{N}=1$

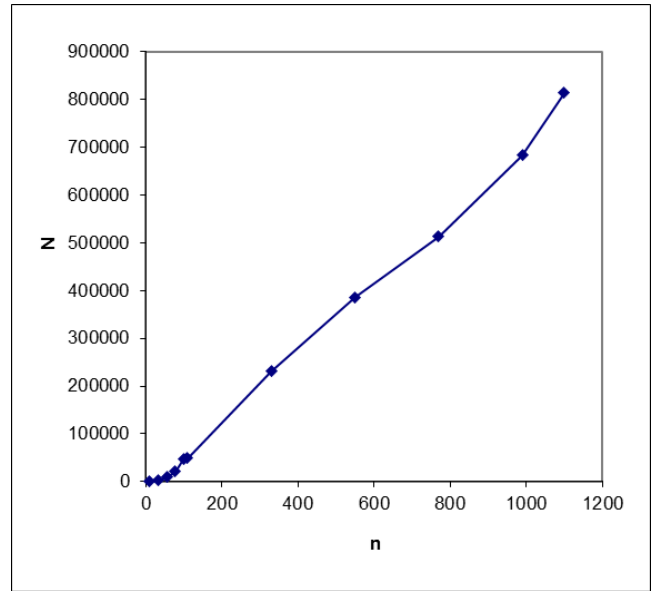

Fig. 7. Relación entre el número de focos de reemisión (N) respecto al número de rayos de reemisión (n), en este caso se ha iniciado la simulación con $\mathrm{N}=81$

El segundo análisis ha consistido en ver qué tipo de relación se puede encontrar cuando analizamos los tres parámetros de valoración claves; el tiempo de simulación, el número de interacciones totales del sistema, y la distribución de energía sobre el plano de detección.

En la figura 8 se ha representado la variación del tiempo de simulación y el número total de interacciones cuando se ha iniciado la simulación con $\mathrm{N}=1$ y en la figura 9 cuando se ha iniciado la simulación con $\mathrm{N}=81$. En la figura 8 se aprecian representaciones de tipo exponencial mientras que en la figura 9 tienden a aproximaciones lineales. Si ahora correlacionamos estos resultados con la distribución de flujo sobre el plano de detección, figuras 10 y 11 para valores iniciales de $\mathrm{N}=1$ y N=81 respectivamente, se aprecia claramente que a medida que incrementa n (columna de la izquierda) la distribución de flujo es más precisa y se puede concluir que un método que modifique únicamente el valor $\mathrm{n}$ (número de rayos de reemisión) es suficiente para determinar el grado de precisión de la simulación y que este grado está directamente relacionado con el tiempo de cálculo que será necesario. 

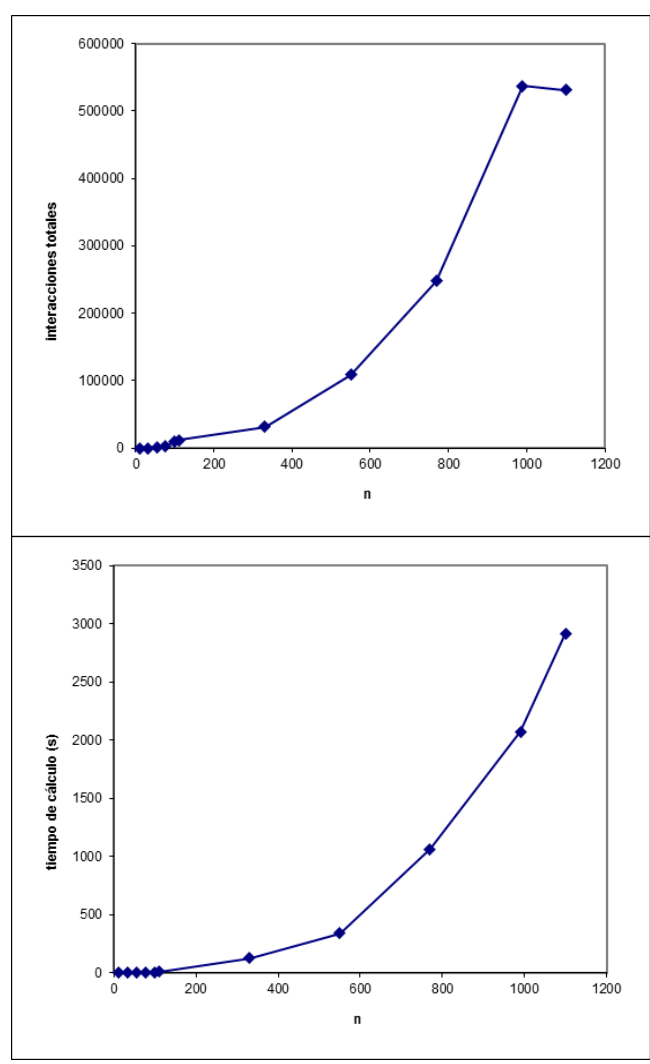

Fig. 8. Relación entre el número de rayos de reemisión (n) respecto a las interacciones totales (arriba) y al tiempo de cálculo (abajo), en este caso se ha iniciado la simulación con $\mathrm{N}=1$

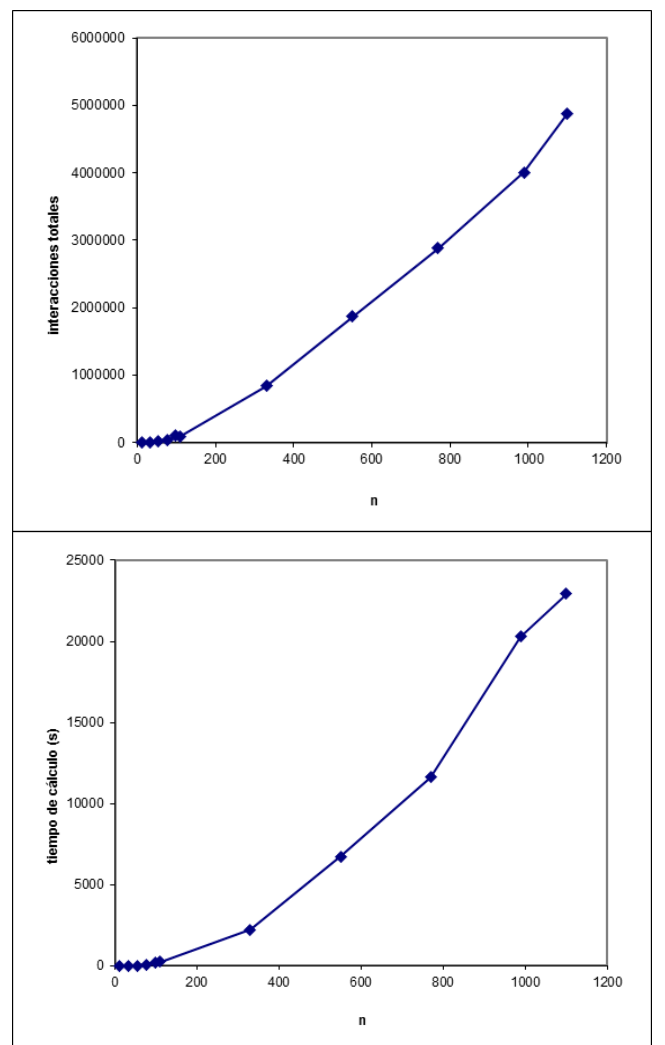

Fig. 9. Relación entre el número de rayos de reemisión (n) respecto a las interacciones totales (arriba) y al tiempo de cálculo (abajo), en este caso se ha iniciado la simulación con N=81. 


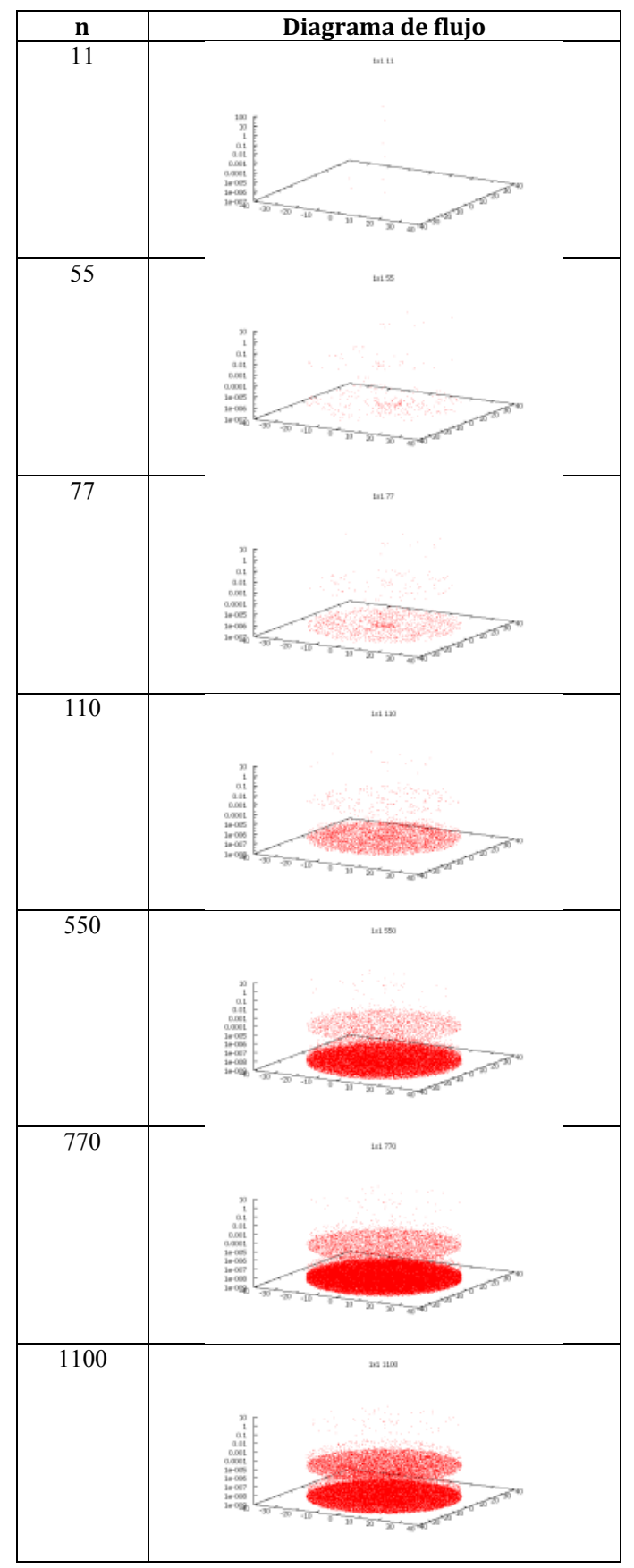

Fig. 10. Relación entre el número de rayos de reemisión (n en la columna de la izquierda) respecto a la distribución de flujo en el plano de detección. En este caso se ha iniciado la simulación con N=1. La escala de flujo es logarítmica. 


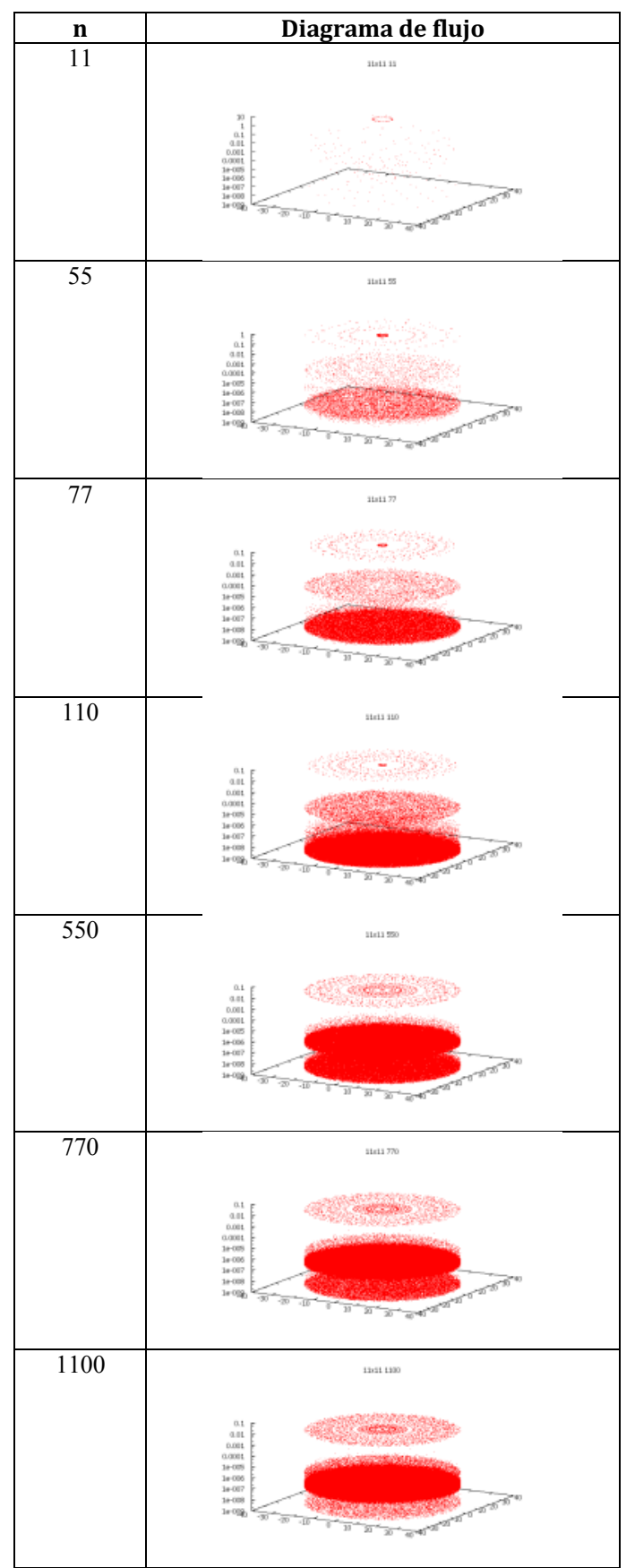

Fig. 11. Relación entre el número de rayos de reemisión (n en la columna de la izquierda) respecto a la distribución de flujo en el plano de detección. En este caso se ha iniciado la simulación con N=81. La escala de flujo es logarítmica.

\section{Conclusiones}

En el presente trabajo se ha analizado la influencia del número de elementos reemisores en el modelado de difusores durante la etapa de optimización. En primer lugar se ha podido ver que modificar o no el número de rayos en que se muestrea la fuente no es suficiente para establecer niveles de calidad o de tiempo de cómputo. El número de rayos de reemisión considerados en la simulación es precisamente el elemento que determina dichos niveles. Esta conclusión afecta a nivel de simulación por lo que es posible aplicarla tanto a la eficiencia en el cálculo de análisis de luz difusa en el ámbito del stray light como en la optimización dual presentada. En este último caso se propone el uso del número de rayos de reemisión como variable para activar/desactivar el uso de funciones de mérito que incluyan el fenómeno de la difusión en la etapa de optimización de diseño óptico. 
El estudio se ha realizado sobre un sistema sencillo compuesto de una fuente de iluminación, una lente biconvexa y una pantalla de detección. Los parámetros objeto del análisis han sido: el tiempo de cómputo por simulación, el número de interacciones totales del sistema y, de forma cualitativa, la distribución de flujo radiométrico que llega a la pantalla de detección.

Se ha constatado que el uso del número de rayos de reemisión es un parámetro que permite utilizar el modelo en diferentes grados de precisión y a su vez controlar el tiempo de cómputo.

Para la inclusión del modelo en un proceso de optimización, el tiempo de cómputo es crítico, siendo el resto de parámetros analizados relevantes en la precisión del modelo. Por lo que se concluye que, considerando los diferentes elementos de reemisión, el número de rayos de reemisión del modelo difusor es un buen candidato para la adaptación dinámica del modelo difusor en su inclusión en un proceso de optimización dual. 